\title{
Cardioprotective effects in aged spontaneously hypertensive rats due to chronic stimulation/activation of sGC without hypotension Emma S Jones ${ }^{1}$, Barbara K Kemp-Harper ${ }^{1}$, Johannes-Peter Stasch ${ }^{2}$, Harald HHW Schmidt*1 and Robert E Widdop ${ }^{1}$
}

\author{
Address: ${ }^{1}$ Department of Pharmacology, Monash University, Australia and ${ }^{2}$ Cardiovascular Research, Bayer HealthCare, Germany \\ Email: Harald HHW Schmidt* - harald.schmidt@med.monash.edu.au \\ * Corresponding author
}

\author{
from 4th International Conference of cGMP Generators, Effectors and Therapeutic Implications \\ Regensburg, Germany. 19-21 June 2009 \\ Published: II August 2009 \\ BMC Pharmacology 2009, 9(SuppI I):P29 doi:I0.II86/I47I-22I0-9-SI-P29
}

This abstract is available from: http://www.biomedcentral.com/I47I-22 I0/9/SI/P29

(c) 2009 Jones et al; licensee BioMed Central Ltd.

\section{Background}

cGMP has recently been suggested to have not only protective but also proliferative and proaggregatory effects. These may differ from nitric oxide, which can also act independently of cGMP. sGC stimulators (which target the reduced $\mathrm{Fe}^{2+}$ form of sGC) and activators (which target the oxidised/heme-free state) are ideal tools to study these as they elevate cGMP in an NO-independent manner. We wanted to investigate the long-term effects of these compounds using the sGC stimulator, BAY 41-2272, and the activator, BAY 58-2667, in aged spontaneously hypertensive rats (SHR). This model of advanced cardiovascular disease exhibits marked cardiac hypertrophy and fibrosis.

\section{Methods}

Aged SHR (20-22 months) were treated for 4 weeks with BAY 41-2272 (1 mg/kg/day, i.p.) or BAY 58-2667 (0.3 mg or $1 \mathrm{mg} / \mathrm{kg} /$ day, i.p.). Mean arterial pressure (MAP) was measured by radiotelemetry and heart:body weight ratio and cardiac fibrosis $(0.001 \%$ Picrosirius red) were determined as indicators of cardiac remodelling.

\section{Results}

MAP was transiently reduced after i.p. injections but basal $\mathrm{MAP}$, measured $\sim 8 \mathrm{hr}$ after injection, remained stable throughout the entire study and was not significantly influenced by drug treatments. Both BAY 41-2272 and BAY 58-2667 dramatically decreased indices of cardiac remodelling such that fibrosis levels were reduced to that of age-matched normotensive Wistar-Kyoto rats (WKY). Cardiac interstitial collagen content (\%) under BAY 412272 was $7.0 \pm 0.3(n=8, P<0.01$ versus vehicle-treated SHR); the high dose of BAY 58-2667, $6.7 \pm 0.2(\mathrm{n}=9, \mathrm{p}<$ 0.001 versus vehicle-treated SHR); vehicle-treated SHR, $9.0 \pm 0.5(n=10)$; vehicle-treated WKY, $6.0 \pm 0.2(n=7)$. Similarly, marked reductions in heart weight: body weight (mg/g) were observed: BAY 41-2272, $4.6 \pm 0.2$; high dose BAY 58-2667, $5.0 \pm 0.2$; vehicle-treated SHR, $5.9 \pm 0.3$; vehicle-treated WKY, $3.5 \pm 0.03$.

\section{Conclusion}

In this model of advanced cardiovascular disease due to aging and hypertension, BAY 41-2272 or BAY 58-2667 rapidly reverses pathological cardiac changes that are already established. Such antihypertrophic and antifibrotic effects occurred without a sustained antihypertensive effect, and indicate that sGC is a valid target for cardioprotection. 